\title{
El Derecho de Familia en la Unión Soviética
}

\author{
Por el Dr. LUDWIK KOS - RABCEWICZ ZUBKOWSKI (*)
}

El Derecho de Familia es un tema tan amplio que no puede ser estudiado, detalladamente, en una sola conferencia. Por eso, me voy a referir, solamente, a las grandes líneas de la historia de las leyes de la Familia en la Unión Soviética.

Lo que nos interesa, no es sólo el hecho de que estas leyes sean aplicadas a la población de un enorme territorio euro-asiático, sino, sobre todo. la valoración de los resultados de los grandes experimentos hechos por las autoridades soviéticas, en las relaciones mútuas entre los esposos, así como entre padres e hijos.

Vamos a comenzar nuestro estudio por el examen del primer texto legislativo de la República Socialista Federada Soviética de Rusia, después de la Revolución de 1917.

Digo la R.S.F.S.R. porque la Unión Soviética fué solamente constituida en el año 1923, medionte un acto, en la forma de tratado de estado, el 30 de diciembre de 1922, confirmado por el Décimo Congreso PanRuso de los Soviets. que proclamó el Primer Congreso de los Soviets de la Unión.

La primera Constitución de la Unión Soviética, fue formulada por el Comité Ejecutivo el 6 de julio de 1923 y confirmada el 31 de enero de 1924 por el Segundo Congreso de los Soviets de la Unión Soviética.

El primer Código referente al Registro de Nacimientos, Muertes y Matrimonios, se promulgó en la República Socialista Federada Soviética de Rusia, el 17 de octubre de 1918 (1). Los decretos anteriores, de 1917, en relación al matrimonio civil y la libertad absoluta de divorcio (2), fueron confirmados por este Código. Las relaciones personales de los casados se basaban en los principios de igualdad total entre el hombre y la mujer.

\footnotetext{
(1).-Publicado el 22 de octubre de 1918 (SU. ASFSR, 1918, No 76/77, st. 818).

(2).--Del 18 de diciembre de 1917, publicado el 20 de diciembre de 1917 (S.U., RSFSR, 1917. No 10, st. 101).
} 
Los contrayentes, en el momento del matrimonio, podían adoptar el nombre del marido, el de la esposa o el de ambos. La obligación del sostenimiento de la familia y del marido, y la "mujer era mútua. Se estableció la separación de bienes entre los esposos. La autoridad de los padres se ejercía conjuntamente. El padre no conservaba el predominio, que tenía antes de la Revolución. En caso de diferencia de opinión, entre los esposos, los tribunales debían resolver los litigios.

La idea de que la ley, como un instrumento de dominación de clases, debería desaparecer gradualmente, durante el primer periodo después de la Revolución, dió como hecho que la vida de familia fuera emancipada de toda interferencia del Estado. De otra parte, los partidarios del "amor libre" apoyaron la abolición de la regulación legal de la vida de la familia. La representante de este grupo, Sra. Kollontai, alegaba que, bajo el régimen socialista, la familia no debía existir, pues es el Estado el que debería tomar bajo su control total a la niñez. Para ella, "La alimentación en común elimina la cocina del hogar, kindergardens y guarderías para niños, que alivian a la madre el peso de las tareas del hogar, trasfiriéndolo a la sociedad; la educación de los niños no es un problema familiar, es un problema del Estado y un problema social".

La Sra Kollantai predecía, también, que el amor se convertiria en un acto puramente fisiológico, tan natural y simple como tomar un vaso de agua. Sin embargo, sus ideas, no estabán completamente de acuerdo con la doctrina de Marx.

La siguiente legislación de la República Socialista Federada Soviética de Rusia (R.S.F.S.R.) sobre la Familia fue promulgada en 1926 (3). Las otras Repúblicas Federadas de la Unión Soviética, adoptaron los mismos principios del "Código de la Familia" ruso de 1926. Estos códigos reconocieron los matrimonios de hecho, como si fueran matrimonios leqalmente registrados, lo que significaba que el registro tenía un carácter puIamente declarativo. La ley no se preocupaba de la fidelidad de los cónyuges. La madre tenía derecho de iniciar acción de paternidad ante un tribunal, después del nacimiento de un niño, si su marido no era el padre de esa criatura. El divorcio fue posible a solicitud de uno de los cónyuges solamente, sin notificación a la otra parte. Una ley especial de 18 de noviembre de 1920, legalizó los abortos, lo que fue justificado con argumentos médicos y por dificultades económicas. Se pensó que era preferible legalizar los abortos y no dejar que se realizaran ilegalmente, lo que era más peligroso para la salud de la mujer.

En el caso de hijos de padres desconocidos, el artículo 141 del Código de 1918, permitía establecer la paternidad colectiva cuando la propia madre no supiera. quien era el padre. Todos los presuntos padres, estaban obligados a pagar los gastos del parto y los de alimentos de la madre y el niño. El artículo 32 del Código de 1918, daba un poder discrecional a las

(3)....-Publicado el 19 de noviembre de 1926, vigente desde el $1^{\text {o }}$ de enero de 1927 (S.U.. RSFSR, 1926, No 82, st. 612). 
Cortes de Justicia. La práctica demostró que los tribunales buscaban siempre a la persona con mayores posibilidades económicas. Bajo tales condiciones, la desintegración de la familia, fue un hecho.

El modo de escoger uno de los sospechosos como padre fue ilustrado en una causa llevada al tribunal en 1928. Una mujer había declarado, en su demanda, que durante una reunión de amigos, se había bebido mucho, y ella había tenido relaciones con un tal Vinogradov e, inmediatamente después, con un tal Lebleu. Cuando nació el niño ella pidió al tribunal escoger uno de los dos como el padre, pues ella misma lo ignoraba. E tribunal de primera instancia juzgó que Vinogradov era el padre, y el tribunal provincial confirmó esa sentencia. El Tribunal Supremo de la R.S. F.S.R., revisado el caso, llegó a la conclusión de que la solución del tribunal era buena y declaró: "El tribunal tomó en cuenta el interés del niño, que tiene que ser protegido, y obtener el minimum necesario para mantenerse. En tales circunstancias, es el demandado quien tiene que probar que no es el padre del niño. Incumbe a la madre presentar su solicitud de modo que apareciese quienes eran los posibles padres". El Tribunal parecía tener la opinión de que tenía derecho de escoger al padre y, entonces, incumbía al que había sido escogido probar que, efectivamente, no era el padre. (4).

Ya en 1925 se hizo evidente que el Estado era incapaz de asumir la educación de los niños sin padres o sin familia y de ahí los cambios en las leyes de la materia. Por ejemplo, el Código de 1918 prohibía la adopción. Sin embargo, el de 1926, Ia autorizó, solamente en interés del niño.

De todas maneras se puede decir que ambos códigos, el de 1918 y ei de 1926, se han caracterizado por su tendencia a una libertad e independencia completa de todos los miembros de la familia.

La Constitución de la Unión Soviética, promulgada en 1936, otorgó, en su artículo 14, a las autoridades centrales, el derecho de determinación de los principios de la legislación concerniente al matrimonio y a la familia, poder que esas autoridades no tenían anteriormente. Esto causó una discusión pública, en la cual se expuso diferentes teorías legales sobre la familia. La teoría de la Sra. Kollontai fue rechazada. La desintegración de la familia fue condenada y se consideró a la familia como la "célula básica del organismo social".

Las reformas legales siguieron gradualmente. El decreto de mayo de 1935 aumentó la responsabilidad de los padres por los delitos de los hijos. El decreto del 27 de junio de 1936 prohibió el aborto e introdujo ciertos cambios en los procedimientos de divorcio (5). La Ley de la Familia, del 8 de julio de 1944, suprimió la validez legal del matrimonio de "hecho" y estableció un complicado procedimiento para el divorcio (6). En consecuencia, la fámilia es, desde entonces, más estable y está mejor protegida.

\footnotetext{
(4).- (L. V. Vinogradov y Lebleu, No 358 (1930) Sud. Prak. RSFSR 19).

(5). - (SZ, SSSR, 1936, No 34, st. 309).

(6).-.-(Vedonosti Vekhovnogo Soveta SSSR, 1944, No 37).
} 
Se demostró que se cometían muchos abusos por la facilidad con que el matrimonio "de hecho" era disuelto, también "de hecho". El legislador soviético volvió en 1944, al principio de la ley de 1917. Solamente un matrimonio registrado crea derechos y obligaciones entre el marido y la mujer.

Los matrimonios "de hecho", admitidos antes por los Códigos no eran ya admitidos. Si las personas casadas "de hecho" deseaban que su unión produjera los efectos juridicos del matrimonio, la ley de 1944 ordenaba que fueran inmediatamente declarados en el Registro Civil, indicando desde cuando existía el matrimonio "de hecho". La nueva norma tardó en dar lugar a decisiones de la jurisprudencia. Una campesina pedía su parte en los bienes de una comunidad familiar, parte que le fue negada, porque su matrimonio con un miembro de la comunidad familiar, había sido un matrimonio "de hecho", no registrado civilmente. La mujer fue simplemente informada que tenía el derecho de solicitar una indemnización por el valor del trabajo que había realizado durante el tiempo que había vivido en comunidad (7).

El primer Decreto del 19 de diciembre de 1917 introduce el divorcio:

a) por acuerdo mútuo de los cónyuges, formulado ante el oficial del estado civil (art. 91), quien tiene atribuciones para redactar un acta verificando la veracidad de acuerdo mútuo. (art. 92).

b) por una demanda de divorcio presentada por uno de los cónyuges, ante los tribunales competentes.

Con relcción al divorcio, el artículo 140 del Código de 1926, precisaba: "En el caso de que la petición de divorcio sea sometida por solo uno de los esposos, una copia de la decisión del oficial del registro civil, debe ser enviada al otro esposo, en la dirección indicada en la petición".

Según este Código de 1926, el divorcio no es mós que un estado "de hecho" del cual los únicos jueces son los interesados.

Sin embargo, el decreto del 27 de junio de 1936, exige la presencia de ambos cónyuges en las oficinas del registro civil y la anotáción del divorcio en los pasaportes de los divorciados. La misma ley aumentó los derechos de registro del divorcio a 50 rublos por el primer divorcio y 150 por el segundo y subsiguientes. La pensión alimenticia para los hijos se elevó de un cuarto a la mitad del salario, lo que fue un arma adicional contra los divorcios frivolos.

Mientras que el legislador modificaba su actitud e indicaba que la destrucción de un matrimonio se debía considerar como una cosa seria, los Tribunales establecían procedimientos completamente distintos a los anteriores al año 1930. Un informe hecho en 1935, sobre la actividad de la Corte Suprema de la R.S.F.S.R., relata un caso semejante al de 1929 . La Corte había llegado a la conclusión de que, aunque la bigamia constituye una infracción criminal, esa circunstancia no impedía reconocer validez a un matrimonio "de hecho", después de otro registrado civilmente $y$ que no había sido disuelto por divorcio. En 1935, se trataba de una mujer que pretendía ser la segunda esposa de un hombre que acababa de morir. Al

(7).-- (VARDUCH Gosyan v, agit Gosyan No 48 (1948) 4 sud. SSSR. 24). 
momento de su muerte, el hombre, según los registros civiles, era casado con otra mujer. El tribunal de primera instancia habia juzgado que las dos mujeres tenían derecho a la herencia, dado que la existencia de dos matrimonios en la misma época no debía ser considerada como bigamia. La Corte Suprema declaró que esa conclusión era errónea, y volvió a enviar el caso al Tribunal, diciendo que "el Tribunal debía establecer si el primero de los matrimonios había cesado de existir; no tenía el derecho de reconocer la existencia de un nuevo matrimonio "de hecho" si el registrado existía en la misma época, y debía proporcionar una contestación precisa a la cuestión de saber cuál de las dos tenía derecho a la sucesión del "de cujus" (8).

Lo actitud de los Tribunales en asuntos de divorcio puede ser jlustrada también por el siguiente: Todo auto de divorcio, proveído por la Corte Provincial debe ser inscrito en los registros del estado civil y el divorcio tiene efecto solamente cuando la transcripción ha sido hecha. Esta disposición de la ley fue aplicada en 1949, en un caso en el cual se trataba de varios intereses sociales. Una mujer trataba de establecer que su hijo había sido adoptado por un cierto Bourgaft ya fallecido. Ella quería asegurar a su hijo la herencia de Bourgaft. La Corte Suprema de la URSS comprobó que Bourgaft había conseguido un divorcio antes de morir, pero que no se había cumplido con la transcripción a los registros del estado civil, como lo exige el código. La Corte juzgó, en consecuencia, que hasta su muerte, él había estado casado. La adopción que él había hecho no podía tener efecto, porque había sido hecha sin el consentimiento de su esposa, como lo exige el Código de la Familia (9).

En otro jucio la Corte Provincial de Alma-Ata, y después, la Corte Suprema de Kazakhstan, habían negado el divorcio porque eran de parecer que los esposos podían volver a vivir juntos. La Corte Suprema de la URSS. tenía otra opinión; señaló el hecho de que los esposos habían dejado de vivir juntos desde 1944, habían repartido los bienes comunes $v$ convenido en dejar los niños con el padre (10).

En otro asunto, un hombre y una mujer, de 49 y 50 años, respectivamente, en la fecha de su boda, trataban de divorciarse sosteniendo que, después de casados, habían seguido viviendo separados, y que nunca habían tenido relaciones conyugales. Explicaban el hecho alegando que, después del matrimonio "habían notado una diferencia tan grande en sus caracteres y costumbres, que la vida común era imposible". Los testigos habían confirmado esa incompatibilidad y la Corte Suprema de la URSS se dejó convencer de que un matrimonio anormal no era posible (11).

En el tercer juicio, la Corte Suprema de la República Socialista Soviética Federada de Armenía había concedido un divorcio, y la Corte Suprema de la URSS confirmó esa sentencia. El expediente indicaba que la

\footnotetext{
(8). -(Sokolova v. Sokolskaya (1935) Sov. Yust. 24).

(9).-(Causa Plotnikova (1950) I sots. Zak. 59).

(10).-(1. v. S. No 36 (1946) o Sud. Prak. SSSR 8).

(11).-(Tchakhunachvili $\vee$. Andrievskaya (1946) 9 Sud, Prak. SSSR 7).
} 
vida común había cesado, en efecto, desde 1941, y que el esposo se habia casado de nuevo después de haber conseguido, de los primeros jueces, una sentencia concediendo el divorcio que había solicitado. La Corte Suprema. de la URSS declaró que, en tales circunstancias, se debía comprender que no era posible restablecer la validez del matrimonio precedente. El único hijo fue entregado a la madre (12).

En un cuarto juicio, el divorcio fue también concedido. Se reconoció que los esposos estaban separados en 1942, y que el esposo había fundado nueva familia con otra mujer y que habia tenido dos hijos con ella. La Corte comprobó que el primer matrimonio, desde 1926 hasta 1942 , había sido turbado por graves disputas y querellas (13).

En todos estos casos, en los que el divorcio fue concedido, parece que los esposos se habian separado antes de ser publicada la Ley de 1944, circlinstancia que pudo tener cierta influencia sobre las sentencias de la Corte. Tal conclusión se sigue de una causa, de fecha de 1946, en la cual las relaciones habian continuado entre los esposns, aunque no fueran siempre pacificas (14). El matrimonio había tenido lugar en 1923 y dos hijos habían nacido, en 1924 y 1929. El esposo solicitó el divorcio, diciendo que no era posible continuar la vida común porque se producían siempre peleas por los celos de su esposa. En primera instancia, el esposo consiguió el divorcio, pero la Corte Suprema de la URSS anuló esta sentencia, porque no habia ringún testigo de dichas peleas, con anterioridad a 1941. En realidad éstas habian empezacio en 1943, después que la esposa conoció las infidelidades de su esposo cucundo estaba en el ejército. Después de la guerra, el esposo había vuelto a la casa y habían reiniciado de nuevo su vida común.

Durante los siguientes años, se nota una reticencia más y más grande en otorgar divorcios. En 1949, la Corte Suprema niega un divorcio ya otorgado por una Corte Provincial, porque el demandante, padre de tres hijos menores, tenía la culpa de que las relaciones con la esposa se hicieran difíciles. Efectivamente, el esposo tenía relación con otra mujer. La Corte Suprema declara: "Las relaciones difíciles en el hogar son culpa del demandante y no pueden constituir base para un divorcio". La sentencia de la Corte en esta causa concluyó admitiendo la actitud francamente inmoral del demanclante; el divorcio no puede ser considerado como justamente otorgado" (15).

La Ley de 8 de julio de 1944 introdujo formalidades adicionales en los procedimientos de divorcio. El que lo solicitaba, debía pagar una suma de 100 rublos al presentar la demanda. Los avisos de iniciación de la acción de divorcio, debían ser publicados en un periódico local, por cuenta del interesado. La presencia de ambas partes ante la Corte era obligatoria. El Tribunal del pueblo estaba obligado a determinar las ra.

(12).--(Causa G. y K. (1946) 4 Sud. Prak SSSR 6).

(13). - - (X v . X (1946) 4 Sud. Prak SSSR 6).

(14). - (K v. K. No 36.830 (1946) 9 Sud. Prak. SSSR 8\%,

(15). - (V. v. V. (1949) 4 Sots. Zak. 61). 
zones de la demanda de divorcio y procurar la reconciliación de las partes. En caso de que no hubiera reconciliación, el que solicitó el divorcio debía presentar la demanda de divorcio al Tribunal de Segunda Instancia. En caso de que el Tribunal reconociera la necesidad de la disolución del matrimonio, la oficina del registro civil debía emitir un certificado de disolución, anotarla en los pasaportes de los divorciados y percibir, de una de las dos partes, una suma entre 500 y 2,000 rublos, según la decisión de la Corte.

La Ley de 1944 no mencionaba las causas de divorcio y dejaba a la Corte un poder discrecional de reconocer o no, que las razones alegadas justificaban el divorcio. Generalmente la Corte declaraba disuelto un matrimonio, cuando se acreditaba que las relaciones maritales no existían, que no habían hijos y, muy particularmente, cuando el marido o la mujer. habian creado ya otra familia.

En cuanto a los abortos, la propaganda en contra de ellos principió algunos años después de la revolución. Finalmente, el decreto de 27 de julio de 1936 (16), prohibió los abortos radicalmente. Esto fue acompañado por medidas de orden económico, como el aumento de la ayuda a las parturientas, ayuda a las familias numerosas, aumento del número de casas de maternidad, de guarderías para niños y de kindergartens, así como el aumento de las penas por falta de pago de pensiones alimenticias. Hoy la maternidad es fomentada como una función social importante. A la madre de diez hijos, que los educa, se le otorga el título de "Madre Heróica" y obtiene una medalla con tal inscripción. Una madre de siete o nueve hijos, obtiene la Medalla "A la Gloria de las Madres"; una madre con cinco o seis hijos, obtiene la Medalla de "La Maternidad". Las madres que obtienen estas medallas reciben una subvención del Estado y, a partir Ce la Lev del 8 de julio de 1944, tal ayuda es otorgada también por el tercer y cuarto hijo. Ninguna diferencia existe entre los hijos legítimos e ilegítimos para el otorgamiento de estas medallas. Hay que señalar que la ley de 1944, abolió el derecho de la madre de intentar una acción de paternidad y pensión alimenticia para el hijo nacido de un hombre con el cual la madre no vive en "matrimonio registrado"; y que una ley de 1956 permite, nuevamente, los abortos, bajo cierias condiciones (17).

(16).--(SZ, SSSR, 1936, No 34, st. 309).

(17).- Aborto: (U. 5-VIII-1954. VVS 17-VIH-1954). Deroga el artículo 4 de la Resolución del Comité Central Ejecutivo de la URSS, del 27 de junio de 1936 que establecía la responsabilidad penal de la mujer por aborto.

(U. 23-XI-1955, V.V.S. 29-VI-1955), Deroga la prohibición de la práctica del aborto, reglamentándola en el sentido de establecer centros médicos especialmente autorizados' for el Ministerio de Salud para tal objeto, así como la responsabilidad penal de las personrs que, sin tener título de médico. intervengan en su práctica fuera de las maternidades $Y$ demás institutos legalmente autorizados.

(L. 28-XII-1955, V. V. S. 14-I-1956). Confirma el Decreto del Presidium del Soviet Supremo de 23 de noviembre de 1955, sobre la derogación de la prohibición del aborto. 
En lo que se refiere a las relaciones entre padres e hijos, el decreto del 31 de mayo de 1935 puso a los hijos bajo la supervisión paterna aumentando también las responsabilidades de los padres por los delitos de los hijos. Mencionamos también que el Código Penal Soviético ha establecido una responsabilidad de toda la familia en los casos de traición y ha permitido el embargo de la propiedad familiar en los casos de fraude de uno de los miembros de la familia.

Entre las razones de la revisión de las leyes de la familia, las demográficas han tenido la mayor importancia. El abuso de los divorcios y abortos ligado a las dificultades económicas, causó una disminución sensible en los nacimientos. Sin embargo, el gran programa de industrialización y de la preparación para la guerra, exigía el aumento del incremento de la natalidad. La prohibición del aborto y las recompensas a las madres de familias numerosas, fueron aplicadas como paliativos naturales, pero esto era insuficiente, era también necesario reconstituir la familia normal y restablecer su vida moral. La delincuencia juvenil y la formación de pandillas tomó proporciones catastróficas. Para contrarrestar esto se aumentó las responsabilidades de los padres por los delitos de los hijos.

La postura de 1918, que prohibía la adopción, bajo el pretexto de que tal adopción disimulaba la obtención de un trabajador más en beneficio de los adoptantes, fue abandonada en 1926, con el objeto de encontrar padres para el gran número de niños huérfanos abandonados, organizados en pandillas ante las cuales la autoridad era impotente. Pero se alegaba, que la adopcion podía servir para eludir las limitaciones del derecho de sucesión.

Con la abolición, en 1926, de las disposiciones del código civil que limitaban el derecho de sucesión, desapareció una de las razones contra la adopción, y la institución fue restablecida, aunque con ciertas precauciones. Sólo los menores de 18 años podían ser adoptados, y únicamente con el consentimiento de los padres. Ese consentimiento no es exigido del padre que ha perdido la patria potestad. El niño que tiene más de diez años, debe también dar su consentimiento. No todos pueden adoptar a un niño, sino únicamente los que tienen las cualidades exigidas para ser nombrados tutores. La ley excluye así varias categorias de personas: los que están bajo curatela, los que tienen intereses contrarios a los del niño y los que han perdido los derechos cívicos. Si, después de la adopción, se descubre que el nuevo estado del niño es perjudicial para él, se puede pedir al Tribunal la revocación de la adopción. La solicitud de adopción tiene que ser aprobada por el organismo público local de tutela y la adopción tiene que ser anotada en el registro civil.

El adoptado tiene todos los derechos y obligaciones del hijo, inclusive el derecho de sucesión y el de alimentos durante su minoría, y aún después, en caso de que no pueda trabajar.

El 1928, el Código fue modificado para establecer una institución con menos consecuencias que la adopción, pero que proporciona al niño sin hogar una protección legal. Esa institución fue llamada "relación de 
dependencia" (18). La condición del niño, en tal relación, es distinta $\propto$ la del adoptado, cuando se trata, por ejemplo, del cambio de apellidos, los derechos de sucesión y la obligación alimenticia. La "relación de dependencia" proporciona menos protección que la adopción, pero más que la tutela. Si los padres del niño mueren o no pueden mantenerlo, la personq que lo acogió, estableciendo esa relación de dependencia, debe seguir participando a su subsistencia y educación, durante su minoría o incapacidad.

Una menor que está en relación de dependencia con una persona, tiene, igualmente, derechos en la sucesión de ésta, si muere sin testamento. Como lo indica el Código Civil, si una persona probadamente incapaz de trabajar y que, de otra parte, estaba completamente bajo la dependencia y cargo del "de cujus" durante un año entero antes de la muerte de éste, dicha persona tiene igual derecho a la sucesión que el cónyuge sobreviviente y cada uno de los hijos. La ley de 1928 , establece que si una persona deja de disponer su testamento acerca de una persona menor que está bajo su dependencia, los herederos deben, en proporción a la herencia, proporcionar a esta persona lo necesario para su subsistencia. La ley relativa a la "relación de dependencia", (que es anterior a la propagada contra la criminalidad infantil), estaba en la línea del nuevo programa que trataba de asignar un hogar a los niños abandonados, aun en el caso de que sus benefactores los hubiesen olvidado en su testamento.

El año 1936 trajo otra modificación legal a favor de los niños sin hogar, pues en esa fecha fue introducida en la URSS la institución del "patronato". La idea base de esa institución es la de inducir a las personas que temen asumir obligaciones personales, a acoger en su hogar a niños abandonados. Según la nueva ley, una persona puede, por contrato, recibir en su hogar a un niño, de cinco meses a catorce años. El Ministerio de Salud tiene poder para firmar ese contrato, si el niño es menor de cuatro años, y si es mayor de cuatro años, incumbe al Ministerio de Educación. La conexión establecida, antes de que el niño llegue a los catorce años, debe terminar cuando el niño llega a los dieciséis. En los distritos campesinos, el contrato tiene que ser firmado por el Presidente del Soviet local, obrando como representante del Ministerio de Seguros Sociales o del Fondo de Mutualidad de la Hacienda Colectiva. Según el contrato, el patrón recibe una indemnización mensual del organismo con el cual fue concluido el contrato.

La relación de patronato está regida exclusivamente por el contrato y el niño no tiene ningún derecho de sucesión o alimenticio. Ni siquiera tiene derecho a la herencia de su patrón; si éste llega a morir, no puede sostener el menor que dependía del patrón, pues es dependiente, en realidad, del organismo que concluyó el contrato con el patrón. Sin embargo, el patrón es considerado como tutor y puede ser responsable, en el campo penal, si el niño, durante el tiempo que está bajo su protección, está sin vigilancia o sin recursos.

(18).-El 29 de noviembre de 1928 (SU, RSFSR, No 22, st. 233). 
Antes de la guerra, se adoptaron nuevas disposiciones. La más sorprendente fue la que imponía un impuesto a las personas sin hijos, en 1941; y en 1944, un tributo fue impuesto a las personas que no tenían más de dos hijos. En ambos casos, se estableció excepciones a favor de los estudiantes, enfermos y esposos separados por la guerra. Los estudiosos extranjeros de las cosas soviéticas, han visto en esas disposiciones fiscales un esfuerzo para aumentar el número de nacimientos, con el fin de colmar las pérdidas debidas a la guerra.

Resumiendo, la teoría de la desaparición gradual de la familia se mantuvo, oficialmente, hasta 1935. Según esta teoría, la vida de familia debía continuar, pero sin responsabilidad económica y legal de sus miembros. La familia sería transformada en una asociación libre, unida solamente por la voluntad de sus miembros. Era la teoría clásica de Marx y Engels. Sin embargo, hasta 1935, la teoría de que la familia desaparecería como entidad económico y legal, fue atacada en la Unión Soviética como una desviación demasiado radical, llamada "desviación izquierdista" de la línea oficial del partido.

La nueva campaña ideológica fue acompañada de cambios legislativos tales como el aumento de la responsabilidad de los padres, prohibición de los abortos, recompensas a las madres de familias numerosas, aumento de derechos por registro de divorcios y, en fin, en 1944, por la creación del procedimiento judicial de divorcio.

El Estado trata hoy de formar una conciencia moral y legal de la familia. para desarrollar su estabilidad. En lo que concierne a las relaciones entre padre e hijos, aquellos tienen responsabilidad legal de educar a sus hijos como ciudadanos honestos y sometidos al Estado. La ley de 31 de mayo de 1935, autoriza a la milicia (policía) a aplicar a los padres multas hasta de 200 rublos, en caso del comportamiento incorrecto de los hijos o cuando éstos pertenezcan a pandillas callejeras. La ley de 29 de julio de 1935, establece la responsabilidad civil de los padres por los delitos de los hijos.

En lo relativo a las relaciones entre marido y mujer, ésta tiene la misma posición legal en el hogar que el marido. A partir del Código de 1926, la separación de bienes fue abolida, la mujer posee los bienes que ha llevado al matrimonio $y$ es propietaria, en común, con su esposo, de todos los bienes oblenidos por uno o por ambos cónyuges durante el matrimonio. Según las instrucciones dadas a las oficinas notariales del Estado, el 17 de noviembre de 1939, es necesario obtener el consentimiento del esposo o esposa, en el caso de enajenación de un inmueble adquirido por el otro cónyuge, después del matrimonio, exceptuando el caso de inmuebjes heredados

Con relación al matrimonio y al divorcio, la primera legislación soviética, representaba una concepción de la familia fundada sobre el hecho físico de la descendencia, más que sobre el matrimonio. Esto encontró una expresión en la doctrina de la igualdad absoluta entre los hijos legítimos e ilegítimos. 
El registro civil del matrimonio, introducido en 1917 y confirmado en 1918, fue declarado opcional en 1926. El registro era simplemente una prueba y no constituía, ell sí mismo, un matrimonio. En 1944, sin embargo, se introdujo una reforma aue decia que solamente un matrimonio registrado creaba deberes y obligäciones para los esposos.

Por fin, el divorcio es concedido hoy sólo después de un proceso ante dos instancias judiciales, lo que tiene por objeto combatir la tendencia al aumento progresivo del número de divorcios. Por ejemplo, en 1935, hubo en Moscú, un 38.3\% de divorcios.

Todo esto prueba que el Estado soviético tiene necesidad de una familia sana y estable, como todo otro estado. La conclusión sacada de este experimento es muy sencilla; el Estado no puede jamás reemplazar a la familia, sino que, por el contrario, es el Estado el que se apoya sobre la familia.

El Partido Comunista Soviético ha tenido que reconocer la importancia de la familia y transformar su legislación para reconstituir la familia, desintegrada por la legislación soviética dada, inmediatamente después de la Revolución.

NOTA.-Este es el texto de una conferencia dictada en la Facultad de Derecho de la Pontificia Universidad Católica por el Dt. Ludwik Kos-Rabcewicz Zubkowski, Profesor del Centro de Estudios Eslavos de la Universidad de Montreal (Canadá). - Este Centro fue fundado en 1948 bajo auspicios del Episcopado Canadiense $y$ ha adquirido desde entonces gran importancia.

Los profesores, que eran tres al iniciarse, son hoY veinte: $y$ el Centro posee más de "200 alumnos: publica, además una revista bilinque: "Estudes Slaves et Est-Européennes". 